\title{
ON FINITENESS OF RINGS WITH FINITE MAXIMAL SUBRINGS
}

\author{
Department of Mathematics \\ Brock University \\ st. Catharines, ontario \\ Canada L2S $3 \mathrm{Al}$
}

H.E. BELL and A.A. KLEIN

Sackler Faculty of Exact Sciences
School of Mathematical Sciences
Tel Aviv University
Tel-Aviv, Israel 69978

(Received July 25, 1991 and in revised form January 20, 1992)

ABSTRACT. It is conjectured that every ring with a finite maximal subring is finite. We prove this conjecture for PI-rings.

KEY WORDS AND PHRASES. PI-ring, maximal subring, prime ring, simple ring, radical.

1991 AMS SUBJECT CIASSIFICATION CODES. 16P70, 16P10.

The group $C\left(\mathrm{p}^{\infty}\right), \mathrm{p}$ a prime, is an infinite abelian group all of whose proper subgroups are finite; and the zero ring on $C\left(\mathrm{p}^{\infty}\right)$ is an infinite ring all of whose proper subrings are finite. The question of the existence of a nonabelian infinite group all of whose proper subgroups are finite was known as Schmidt's Problem, and was answered affimatively by Olsanskii [1], who constructed an infinite group in which all proper subgroups are of prime order. The analogous question for non-commutative rings has a negative answer, since Laffey [2] has proved that any infinite ring has an infinite cormutative subring.

Observe that in Olsanskii's example, all the proper subgroups are maximal. The corresponding question for rings - whether there exists an infinite ring all of whose proper subrings are maximal - has a negative answer; indeed, Szele [3] has shown that any ring with both ascending chain condition and descending chain condition on subrings is finite.

The Olsanskii example does, however, suggest an interesting and apparently difficult problem for rings: whether there exists an infinite ring having a finite maximal subring. It was proved by Bell and querriero [4] that a commutative ring with a finite maximal subring is finite, and it is our purpose to extend this result to PI-rings. The full force of the PI assumption is used only once in the proof of our theorem; the proofs of the lemmas use only the fact that the class of PI-rings is closed under taking subrings and homomorphic images. Thus it is not unreasonable to conjecture that any ring with a finite maximal subring must be finite.

In what follows, the center of a ring $R$ is denoted by $Z(R)$. The subring generated by $\mathrm{T}$ is denoted by $\langle\mathrm{T}\rangle$. 
We proceed to consider rings that have a finite maximal subring. We start by recalling a crucial result from [4], which is obtained by applying an interesting result of Lewin [5].

LEMA 1. If the ring $R$ has a finite maximal subring, then $R$ has only finitely many ideals.

As in [4], assume $R$ is an infinite ring with a finite maximal subring $S$ such that $|S|$ is minimal. Of course $|S|>0$, since a ring with no proper subrings is finite. If $L$ is a nonzero ideal of $R$, then $L \nsubseteq S$, for otherwise $S / L$ is a finite maximal subring of the infinite ring $R / L$ and $|S / L|<|S|$, in contradiction of the minimality of $|s|$.

LEMMA 2. (i) If $L$ is a nonzero ideal of $R$, then $L+S=R$.

(ii) $|\mathrm{S}| \mathrm{R}=0$.

PROOF. By the above observation $L \notin S$, so $L+S \not S$; and since $L+S$ is $a$ subring, we get $L+S=R$. For the second part, let $L=\{a \in R|| S \mid a=0\}$. Then $L$ is an ideal of $R$ containing $S$, so $L=R$.

LEMMA 3. (i) $R$ has a minimal nonzero ideal $I$ which is contained in any nonzero ideal of $R$; and as a ring, $I$ is simple.

(ii) $R$ is a prime ring.

PROOF. By Lerma $1, R$ has a minimal nonzero ideal $I$. If $L$ is any nonzero ideal of $R$, then by Lerma $2, L+S=R$; hence $|R / L| \leq|S|$, and in particular $|R / I| \leq|S|$. Now if $I \notin L$, then $I \cap L \varsubsetneqq I$, so $I \cap L=0$ by the minimality of $I$. But this implies that $R$ embeds in the finite ring $(R / I) \oplus(R / L), a$ contradiction. Thus we have $I \subseteq L$.

In proving that $I$ is a simple ring, we first show that $I^{2} \neq 0$. We prove that $I^{2}=0$ implies $R$ is finite. We have $I+S=R$; and since $S$ is not an ideal of $R$, either IS $\notin S$ or SI $\notin S$. Assume IS $\ddagger S$ and let a $\in$ IS $\backslash S$. Since $a \notin S$ we have $R=\langle S, a\rangle$. But $a \in I S \subseteq I$ and $I^{2}=0$, so any product containing the element a twice is 0 ; and therefore $R=S+Z a+a S+S a+S a S$. By Lerma 2 (ii) $\mathrm{za}$ is finite, so $\mathrm{R}$ is finite.

Now since $I^{2} \neq 0$, the left (right) annihilator of $I$ is 0 , for otherwise it is an ideal containing $I$, implying that $I^{2}=0$. It follows that if $L$ is $a$ nonzero ideal of $I$, then $I L \neq 0$ and ILI $\neq 0$. But ILI is an ideal of $R$ contained in $I$, so $I L I=I$; and since ILI $\subseteq L \subseteq I$, we get $L=I$. Thus $I$ is a simple ring.

The ring $R$ is prime, for if $L, K$ are nonzero ideals of $R$, both contain $I$ and hence $L K \supseteq I^{2} \neq 0$. 
Note that $R$ has prime characteristic $p$, and therefore the additive group of $S$ is a finite p-group, so $|S|$ is a power of a prime. In addition, it follows easily from the beginning of the proof of Theorem 8 in [4] that the radical $J(S)$ of $S$ satisfies $J(S) R \subseteq S$ and $R J(S) \subseteq S$. Thus we get $J(S)^{2} R \subseteq J(S) S \subseteq J(S)$, so $J(S)^{2} R$ is a nilpotent right ideal of $R$. But $R$ is prime, so $J(S)^{2} R=0$ and therefore $\mathrm{J}(\mathrm{S})^{2}=0$.

LEMMA 4. The center of $R$ is contained in $S$.

PROOF. We prove that if $z(R) \backslash S \neq \phi$, then $R$ is finite. Let $d \in Z(R) \backslash S$. Since $S$ is a maximal subring, we have $\langle S, d\rangle=R$, and of course $\langle S, Z(R)\rangle=R$. Since $S$ is not an ideal of $R$, we get $S Z(R) \nsubseteq S$, so $R=S+S Z(R)$. This and the primeness of $R$ imply that $S$ is prime; therefore $S=M_{r}(F), F$ a finite field. If $e$ is the identity element of $S$, then $e \in Z(R)$ since $d \in Z(R)$. It follows that $e R$ is an ideal of $R$ and $e R \supseteq e S=S$, so $e R=R$; and this implies $e$ is the identity element of $R$. Using again the assumption that $d \in Z(R)$, we get that $R$ $=S[d]$ is the ring of polynomial expressions in $d$ with coefficients in $S$; thus we have $R=S[d]=M_{r}(F)[d]=M_{r}(F[d])$. But since $S$ is a maximal subring of $R$, $d$ must be algebraic over $F$, so $F[d]$ is a finite field and $R$ is finite.

we could restrict the above considerations to PI-rings without affecting any of the results given. (By a PI-ring we mean, as in [6, p. 88], a ring which satisfies a polynomial identity with coefficients in $\mathbb{Z}$, one of which is 1. ) With this observation we are ready to prove:

THEOREM. If $R$ is a PI-ring with a finite maximal subring $S$, then $R$ is finite.

PROOF. Assuming the result is wrong, choose a counterexample with $|S|$ minimal. Let $I$ be the minimal ideal of $R$ as in Lerma 3 . Since $R=I+S$ and $R$ is infinite, $I$ is also infinite. Since $R$ is PI, the subring $I$ is also PI; and being simple, it is finite dimensional over its center $Z(I)[6, p .98]$. Thus $Z(I)$ is infinite. Since $R$ is prime, $z(R) \supseteq Z(I)$; thus $z(R)$ is not contained in $S$, in contradiction of Lerma 4 . This proves that $R$ is finite.

In conclusion, we note that if the conjecture stated in the introduction is false, our results provide a great deal of information about certain counterexamples. There must be a counterexample which, as Olsanskil's example would suggest, has a rather simple subring and ideal structure but is also badly non-commutative.

ACKNOWLEDGEMENT. H. E. Bell was supported by the Natural Sciences and Engineering Research Council of Canada, Grant No. A3961. 
REFERENCES

1. OLSANSKII, A. Infinite groups with cyclic subgroups, Soviet Math. Dokl. 20 (1979), 343-346.

2. LAFFEY, T. J. On commutative subrings of infinite rings, Bull. London Math. Soc. 4 (1972), 3-5.

3. SZELE, T. On a finiteness condition for modules, publ. Math. Debrecen $\underline{3}$ (1954), 253-256.

4. BELL, H. E. and GUERRIERO, F. Some conditions for finiteness and commutativity of rings, Internat. J. Math. Math. Sci. 13 (1990), 535-544.

5. LEWIN, J. Subrings of finite index in finitely generated rings, J. Algebra $\underline{5}$ (1967), 84-88.

6. ROWEN, L. H. Ring Theory II, Academic Press, 1988. 


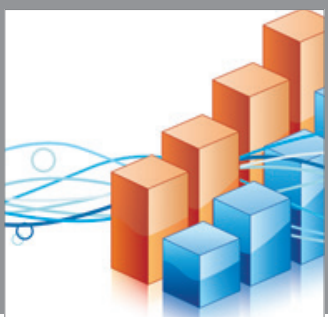

Advances in

Operations Research

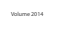

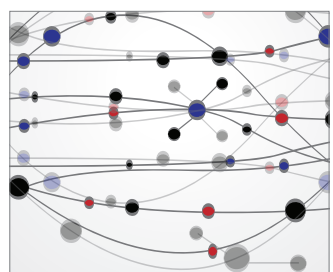

\section{The Scientific} World Journal
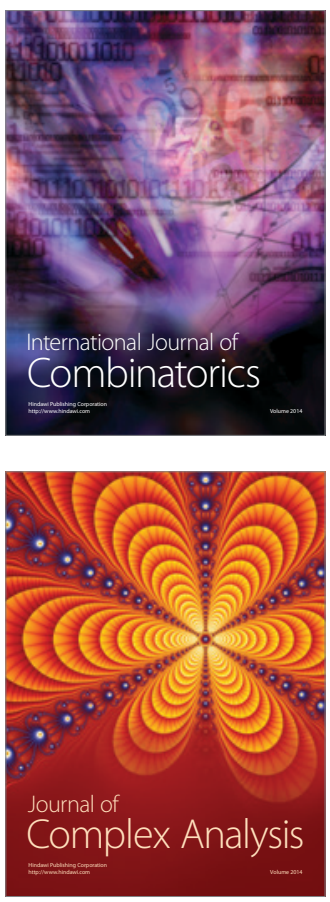

International Journal of

Mathematics and

Mathematical

Sciences
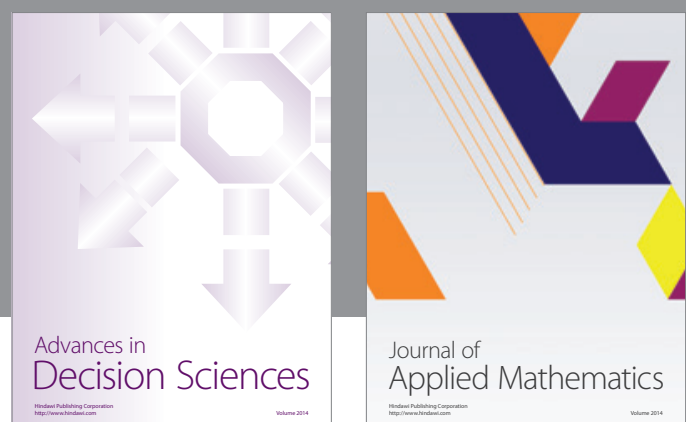

Journal of

Applied Mathematics
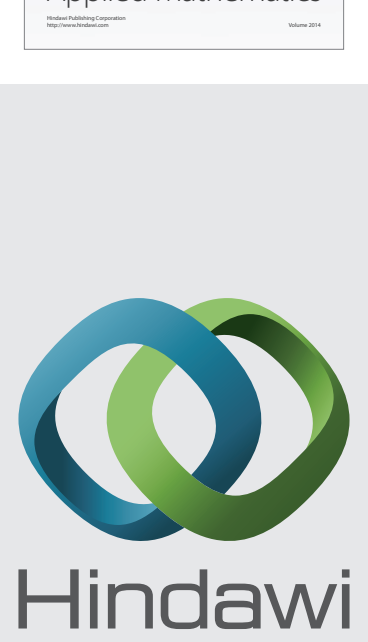

Submit your manuscripts at http://www.hindawi.com
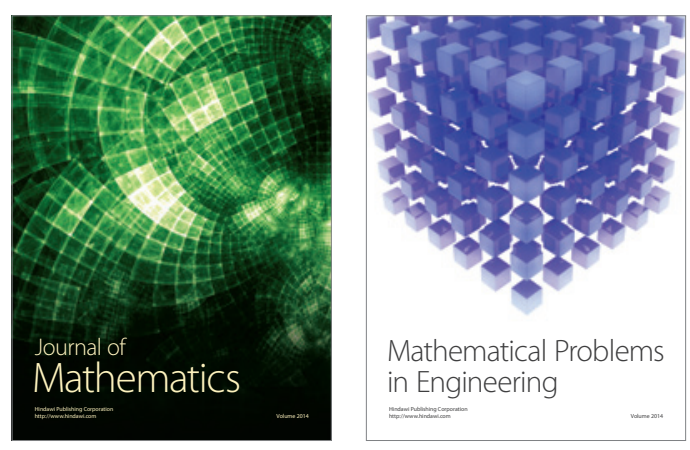

Mathematical Problems in Engineering
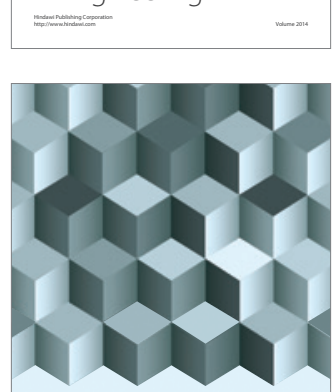

Journal of

Function Spaces
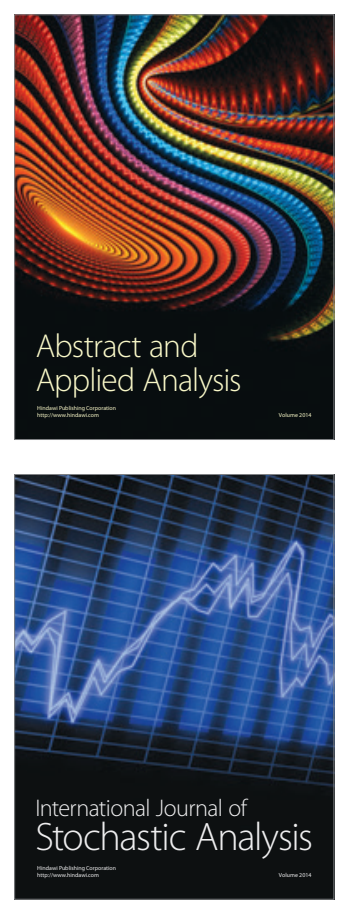

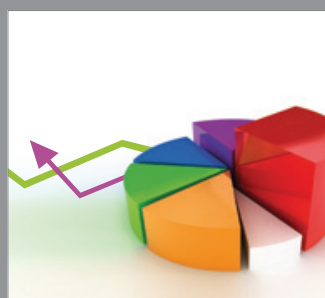

ournal of

Probability and Statistics

Promensencen
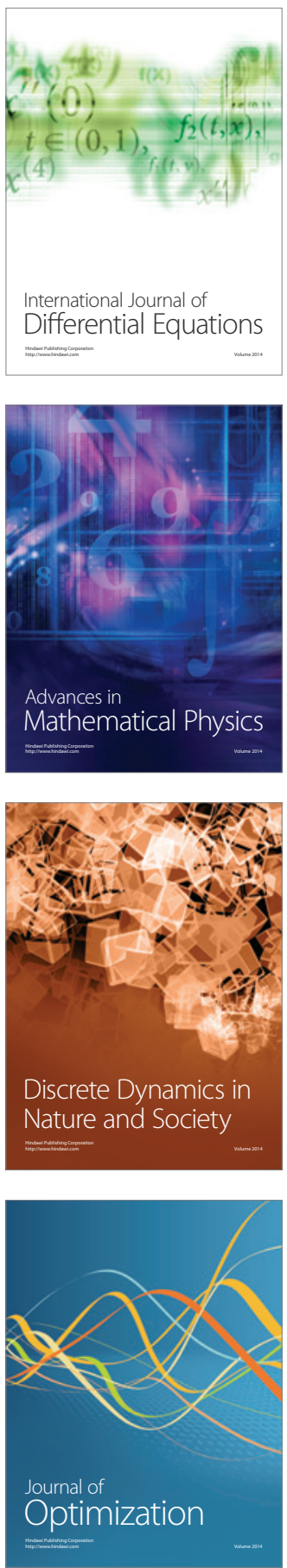